\title{
Cardiac Autonomic Responses and Adaptation to Repeated Bouts of Eccentric Exercise
}

\author{
Choun-Sub Kim PhD, Min-Ghyu Sun MS, Maeng-Kyu Kim PhD \\ Sports Medicine Lab., Department of Physical Education, Kyungpook National University, Daegu, Korea
}

PURPOSE: The current study was performed to examine the effects of acute eccentric resistance exercise (ERE) on heart rate variability (HRV) and to determine whether cardiac autonomic regulation adapts to the repeated bout effect.

METHODS: Isokinetic eccentric exercise consisted of five sets of six maximal contractions of the knee extensor with an angular velocity of $90 \%$ second. College-aged men $(n=11)$ underwent two sessions of ERE with a 3-week interval. Muscle damage indicators such as the range of motion (ROM) and muscle echo intensity (EI) were measured. The post-exercise cardiac autonomic response was assessed by spectral analysis of HRV. Low frequency (LF) and high frequency (HF) bands, the normalized unit (nu) for each band power, and the LF-to-HF (LF/HF) ratio were determined. All variables were recorded at 24, 48, 72, and 96 hours post-exercise as well as before and immediately after ERE.

RESULTS: The EI and ROM of the rectus femoris changed significantly after the maximal ERE. There was a significant interaction effect between the time and bout for EI. Significant changes in LF and HF nu were observed at 72 (1st ERE) and 96 hours (2nd ERE) after each ERE. The LF/HF ratio was also significantly increased at 24 (1st ERE) and 72 hours (2nd ERE) after each ERE. However, no interaction effects on changes in the HRV indices were observed.

CONCLUSIONS: The present study suggests that acute ERE leads to cardiac autonomic imbalance for a sustained period and that repeated bouts of ERE cannot alleviate cardiac autonomic responses to exercise.

Key words: Eccentric resistance exercise, Cardiac autonomic imbalance, Heart rate variability, Exercise-induced muscle damage, Repeated bout effect

\section{INTRODUCTION}

It is a well-known fact that participating in systemically designed exercise training provides critical benefits that prevent premature mortality due to cardiovascular diseases [1]. Resistance training may also enhance heart rate variability (HRV), reflecting cardiac autonomic regulation (CAR) in patients with cardiac autonomic imbalance [2]. However, controversies remain regarding the cardio-protective effects of regular physical activity, in particular resistance training. For instance, a previous study investigating the effects of 12 -week isokinetic eccentric strength training in healthy older men indicated a significant increase in the peak torques of the quadriceps and hamstring muscle groups [3]. However contrary to their hypothesis, the authors were faced with an unexpected result. Eccentric resistance training led to a decrease in HRV, and further, a physiological mechanism that explains eccentric training-induced cardiac autonomic imbalance has not been identified to date [3].

The effects of exercise training may be attributed to physiological responses to a single session of exercise and post-exercise hypotension was found to contribute to the improvement of the hypertension induced by exercise training in a previous study [4]. The eccentric resistance exercise

Corresponding author: Maeng-Kyu Kim Tel +82-53-950-5937 Fax+82-53-955-4754 E-mail kimmkaknu.ac.kr

*This thesis is partially revised from Kim Choun-Sub's doctoral thesis.

*This work was also supported by the National Research Foundation of Korea Grant funded by the Korean Government (NRF-2017R1C1B1006196).

Keywords Eccentric resistance exercise, Cardiac autonomic imbalance, Heart rate variability, Exercise-induced muscle damage, Repeated bout effect

Received 10 Jul 2020 Revised 24 Sep 2020 Accepted 9 Oct 2020

(a) This is an Open Access article distributed under the terms of the Creative Commons Attribution Non-Commercial License (https://creativecommons.org/licenses/by-nc/4.0/) which permits unrestricted non-commercial use distribution, and reproduction in any medium, provided the original work is properly cited. 
(ERE) employed in the above-mentioned study consisted of muscle movements, which generate force during active lengthening contraction [5]. As eccentric exercise (ECC) can generate a high degree of force with low metabolic demand, exercise modalities involving eccentric muscle contractions are popular with both athletes and elderly people [6]. However, ECC leads to exercise-induced muscle damage (EIMD) [7]. A single session of ECC has been employed as a conventional tool in an experimental design for creating EIMD, especially in muscle groups unaccustomed to ECC [8]. Typically, unaccustomed ECC may provoke symptoms of muscle damage, such as reduced muscle force and range of motion (ROM), the emergence of delayed onset muscle soreness (DOMS), and increased muscle swelling, as well as increased muscle echo intensity (EI) [9]. Interestingly, CAR or HRV indices also are likely to depress by an inflammatory response which is postulated to cause increases of muscle swelling and muscle echo intensity [10].

On the other hand, the initial bout of ECC can have a protective effect against muscle damage from a subsequent bout, and thus EIMD caused by eccentric muscle contraction would be offset via repeated bouts of ECC, a process known as the "repeated bout effect (RBE)" [7]. To date, however, there is no clinical information on cardiac autonomic responses to a single bout of ERE and whether the RBE can induce cardiovascular adaptations. Whether acute ECC contributes to cardiac autonomic imbalance is an urgent question that needs to be answered, given the dramatic disruption of cardiovascular homeostasis by exercise as well as the incidence of cardiac events such as sudden death after exercise [11]. Therefore, the present study was conducted to investigate changes in HRV indices as well as muscle damage indicators following maximal ERE and to determine whether cardiac autonomic responses adapt to repeated bouts of ECC.

\section{METHODS}

\section{Participants}

Since exercise training may provide protection against EIMD, physically inactive college-aged men who had not exercised regularly for at least 12 months were eligible for inclusion. The main exclusion criteria were as follows: (1) Known cardiovascular and metabolic problems, (2) taking prescribed medications, (3) taking any anti-inflammatory drugs (e.g., non-steroid anti-inflammatory drugs) or nutritional supplements (e.g., vitamins and amino acids) for at least 2 weeks before participating in the 1st ERE, and (4) hypertension (systolic blood pressure $>140 \mathrm{~mm}$
$\mathrm{Hg}$ or diastolic blood pressure $>90 \mathrm{~mm} \mathrm{Hg}$ ) and/or obesity (BMI $>30 \mathrm{~kg} /$ $\left.\mathrm{m}^{2}\right)$.

Sample size analysis was performed by using the $\mathrm{G}^{*}$ power 3.1.9.2 software (Heinrich-Heine-Universität Düsseldorf, Düsseldorf, Germany; http://www.gpower.hhu.de/) for analysis of variance (ANOVA) [12]. To calculate total sample size presented by Cohen (effect size $=0.25)$, the following variables were set in repeated measures, within-between interaction, and ANOVA approach: i) a err prob (the probability for type I error $)=0.05$, ii) Power $(1-\beta$ err prob; type $\mathbb{I}$ error $)=0.8$, iii) Number of groups $=2$ (first and second bout), and iv) Number of measurements $=6$ (pre, post, 24 hour, 48 hour, 72 hour, and 96 hour after ERE bout).

After excluding individuals who dropped out $(\mathrm{n}=1)$ or had an abnormal pattern in muscle EI $(n=1)$, data for 11 men were included in statistical analysis and the study was performed with a randomized controlled design.

The present study was approved by the Institutional Review Board (IRB) of Kyungpook National University [2018-0067] and all experimental procedures strictly followed the Declaration of Helsinki.

\section{Study design}

All participants in this study performed the 2nd ERE after a 3-week wash-out period following the 1st ERE. All of the data were collected before and immediately after as well as 24, 48, 72, and 96 hours after both ERE bouts. Experimental procedure and measuring sequence are presented in Fig. 1. Since several measuring techniques such as evaluation of muscle pain using pressure algometer may have an acute influences on CAR, r-r intervals at rest were always recorded first. Moreover, maximum voluntary isometric contraction torque which can perturb the results of all measuring variables was tested last.

For at least 24 hours before each experimental trial and up to 96 hours after ERE, all participants were instructed to abstain from taking any drugs or receiving treatment that would affect the exercised muscles and to avoid alcohol and caffeine use as well as strenuous physical activity.

\section{Familiarization session}

Anthropometric and hemodynamic variables were evaluated at least 3-5 days before each experimental session. During the familiarization session, all participants became accustomed to ERE performed on an isokinetic dynamometer (HUMAC NORM, CSMi Medical Solutions, Stoughton, MA). The isokinetic ECC for each pre-practice was performed with the angular velocity $(9 \%$ second) used to return the knee 


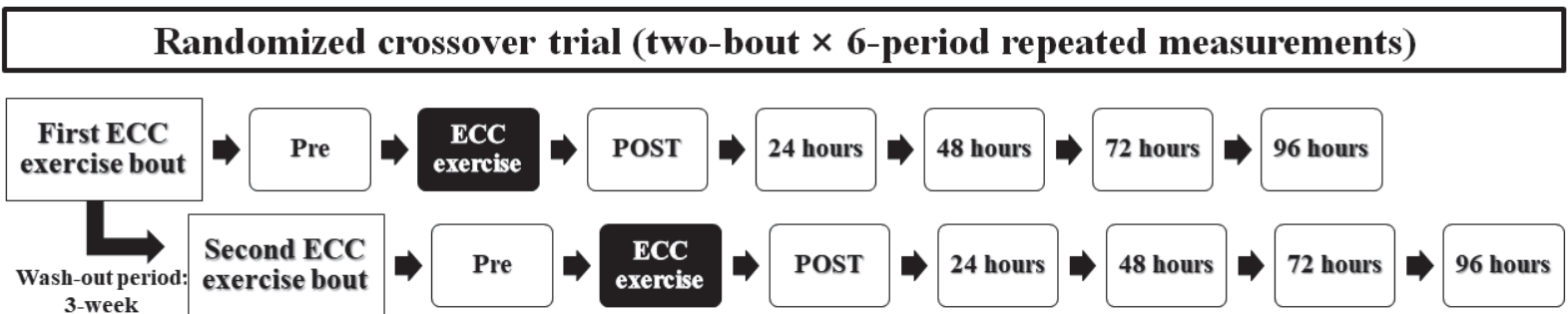

Dependent Variables

Heart rate variability $\rightarrow$ Muscle soreness $\rightarrow$ Limb circumference $\rightarrow$ Range of motion $\rightarrow$ Ultrasound imaging $\rightarrow$

Maximal voluntary isometric contraction torque

Fig. 1. Experimental procedure.

joint to the extension position during the maximal ERE.

\section{Eccentric exercise protocol}

Repeated bouts of ERE consisted of the maximal isokinetic ECC, which was performed using the knee extensor identified as the nondominant limb. Each bout of isokinetic ECC consisted of five sets of six maximal contractions with an angular velocity of $90 \%$ second, and each of the contractions was performed within a ROM between anatomical zero and $90^{\circ}$ [9]. The isokinetic dynamometer was programmed to return to anatomical zero at an angular velocity of $9 \%$ second with a 10 -second rest period between eccentric contractions, and the rest period between sets was set at 2 minutes [9].

\section{Dependent variables}

1) Muscle El

A 7.5-MHz linear array ultrasound probe attached to a Logiq 200 PRO system (GE Medical Systems, Milwaukee, USA) was placed on the thigh to obtain a B-mode image of the rectus femoris muscle with a constant angle and pressure throughout the measurement period. To evaluate the muscle EI, the transverse image of the rectus femoris muscle was scanned at $50 \%$ of the distance between the lateral condyle of the femur and the great trochanter [13]. The measurement point was marked on the dermal surface to ensure consistent placement of the ultrasound probe, and all B-scan images were collected by the same trained examiner. The collected ultrasound images were stored on a laptop running ImageJ software (Version 1.52a, National Institutes of Health, Bethesda, Maryland), and the muscle EI was calculated using a gray-scale histogram $(0=$ black and $256=$ white $)$ for the region of interest $($ ROI: $1 \times 1=1$ $\left.\mathrm{cm}^{2}\right)[14]$.
2) $\mathrm{ROM}$

The knee joint angles for voluntary maximal flexion (FANG) and maximal extension (EANG) were measured using a plastic goniometer (Baseline, Fabrication Enterprises Inc., White Plains, NY). EANG was determined when the participant performed maximal extension of the knee joint, while FANG was measured as the participant attempted to touch their heel to their buttock. The knee joint ROM was calculated by subtracting FANG from EANG [9].

\section{3) MVIC torque}

MVIC torques of the quadriceps muscle were evaluated using the isokinetic dynamometer and determined as the highest values of two maximal contractions performed to generate maximal force at a knee joint angle of $90^{\circ}$ for 3 seconds, with a 45 seconds, rest period between trials [9].

\section{4) Thigh circumference}

Thigh circumference (TC) was measured using a tape measure at the mid-trochanterion tibiale laterale site perpendicular to the longitudinal axis while the subject was standing [15].

\section{5) DOMS}

To evaluate muscle soreness, a hand-held dial pressure algometer device (Baseline Push Pull Force Gauge Model 12-0304; Fabrication Enterprises Inc., USA) equipped with a flat circular probe $\left(1.52 \mathrm{~cm}^{2}\right)$ was utilized. Participants were instructed to indicate the muscle pain perceived at a pressure of $4 \mathrm{~kg} / \mathrm{second}$ on a visual analog scale consisting of a 100$\mathrm{mm}$ line representing "no pain" at one end $(0 \mathrm{~mm})$ and "very, very painful" at the other $(100 \mathrm{~mm})[16]$. 


\section{6) HRV}

To trace the cardiac autonomic responses following repeated bouts of ERE, successive r-r intervals were collected for more than 10 minutes at rest in a sitting position via the Polar RS800CX heart rate monitor (Polar Electro Oy, Kempele, Finland) with a sampling rate of 1,000 Hz. A shortterm power spectrum analysis of HRV was conducted, which required successive r-r intervals of more than 5 minutes [17]. The raw data were extracted as a text file (.hrm) using the Pro-trainer Polar 5 software (version 5.40.171, Polar Electro, Finland), and then transferred to the HRV analysis software (premium version 3.0.1, 2017, Biosignal Analysis and Medical Imaging Group, University of Kuopio, Finland, MATLAB) [18]. To obtain low frequency (LF, 0.04-0.15 Hz) and high frequency (HF, $0.15-0.40 \mathrm{~Hz}$ ) band power, the power spectral density was calculated with fast Fourier transform. The normalized value of the LF component was used to assess the sympathetic efferent activity, while the normalized unit of the HF component was considered to reflect the dominant parasympathetic influence. The ratio between the LF and HF band powers (LF/HF ratio) was utilized as an indicator of sympathovagal balance.

\section{Statistical analysis}

All data were presented as the mean \pm standard error of the mean (SEM) using the SPSS software package (Statistical Package for the Social Sciences Software, Version 25.0, Chicago, IL, USA). Normality of sample distribution was identified via Shapiro-Wilk test and Levene's test was employed to confirm homogeneity of variance. Changes in each dependent variable over time were analyzed with one-way ANOVA. When a significant time effect was found, a Bonferroni post hoc test was performed to identify significant differences from the baseline value. Repeated-measures ANOVA was employed to identify interaction effects. If a significant interaction effect was found, a Wilcoxon signed-rank test was performed to identify significant differences between bouts for each of the time points. The level of significance was set at 0.05 .

\section{RESULTS}

Although the maximal voluntary isometric contraction (MVIC) torque was significantly greater at baseline for the 2nd ERE than for the 1st ERE, there were no differences in the baseline values between the two experimental sessions with regard to the clinical characteristics and other dependent variables (Table 1).
Table 1. Comparison of characteristic in participants between first- and repeated bouts of eccentric exercise at baseline

\begin{tabular}{lrcc}
\hline Variables & \multicolumn{1}{c}{ 1st ERE } & 2nd ERE & $p$ \\
\hline Characteristics & & & \\
Age $(\mathrm{yr})$ & $23.45 \pm 0.80$ & - & \\
BMI (kg/m ${ }^{2}$ ) & $23.83 \pm 0.87$ & $23.67 \pm 0.83$ & .328 \\
Percent body fat (\%) & $16.7 \pm 1.68$ & $16.54 \pm 1.71$ & .722 \\
SBP (mmHg) & $119.64 \pm 2.66$ & $116.82 \pm 2.02$ & .372 \\
DBP (mmHg) & $73.09 \pm 2.09$ & $68.64 \pm 1.25$ & .059 \\
MAP (mmHg) & $88.61 \pm 2.10$ & $84.7 \pm 0.80$ & .062 \\
Mean HR (beats/min) & $66.09 \pm 1.81$ & $66.82 \pm 3.54$ & .894 \\
Muscle damage & & & \\
RF El (a.u.) & $37.88 \pm 1.41$ & $36.86 \pm 1.91$ & .790 \\
ROM (degrees) & $109.73 \pm 2.30$ & $110.77 \pm 1.81$ & .220 \\
MVIC torque (Nm) & $203.27 \pm 16.41$ & $234.27 \pm 15.21$ & .008 \\
Thigh circumference (cm) & $53.72 \pm 1.34$ & $53.55 \pm 1.32$ & .888 \\
Muscle soreness (cm) & $1.18 \pm 0.38$ & $1.00 \pm 0.27$ & .441 \\
Heart rate variability & & & \\
LF nu & $54.1 \pm 5.94$ & $53.57 \pm 5.05$ & .790 \\
HF nu & $45.83 \pm 5.93$ & $46.39 \pm 5.05$ & .790 \\
LF/HF ratio & $1.76 \pm 0.53$ & $1.49 \pm 0.32$ & .445 \\
\hline
\end{tabular}

Values are means \pm SEM.

ERE, eccentric resistance exercise; BMI, body mass index; SBP, systolic blood pressure; DBP, diastolic blood pressure; MAP, mean arterial pressure; $\mathrm{HR}$; heart rate; RF, rectus femoris muscle; El, echo intensity; ROM, range of motion; MVIC, maximal voluntary isometric contraction; LF nu, normalized low frequency $(0.04-0.15 \mathrm{~Hz})$; HF nu, normalized low frequency (0.15-0.4 $\mathrm{Hz}$ ); LF/HF ratio, ratio between LF and HF band powers. $p$-values are calculated by Wilcoxon signed-rank test.

In each ERE, although the MVICs decreased sharply immediately after exercise and gradually increased during the recovery periods, there were no significant differences during the two sessions $(p>.05$, respectively) (Table 2). TC and muscle soreness increased after exercise and decreased over time after all ERE bouts, but both indicators had no statistical effects within each experimental session ( $p>.05$, respectively) (Table 2).

The muscle EI of the rectus femoris changed significantly during all experimental sessions (1st ERE, $p<.05$; 2nd ERE, $p<.01$ ) (Fig. 2A). The EI increased more significantly after the 1st ERE than after the 2nd ERE and significant interaction effects ( $p=.041)$ were found (Fig. 2A). Moreover, there was a statistical difference between bouts at 72 hours after ERE $(p<.05)$ (Fig. 2A). Although the changes in ROM decreased more significantly after the 1st ERE than after the 2nd ERE, there was no significant interaction effect ( $p=.337)$ between time and bout (Fig. 2B). However, the change in the ROM following the 1st ERE was statistically significant $(p<.01)$, but there were no statistical differences in the ROM change after the 2nd ERE ( $p>.05$ ) (Fig. 2B).

The changes in LF nu from baseline exhibited similar patterns after 
Table 2. Changes of muscle damage indicator during repeated bouts of eccentric exercise

\begin{tabular}{|c|c|c|c|c|c|c|c|c|}
\hline Variables & & Pre & Post & 24 hours & 48 hours & 72 hours & 96 hours & $p$ \\
\hline \multirow[t]{2}{*}{ MVIC torque (Nm) } & 1st ERE & $203.27 \pm 16.41$ & $177.64 \pm 8.99$ & $190.36 \pm 8.69$ & $205.36 \pm 9.17$ & $228.91 \pm 15.54$ & $218.91 \pm 13.35$ & .063 \\
\hline & 2nd ERE & $234.27 \pm 51.21$ & $219.09 \pm 12.52$ & $222.09 \pm 13.55$ & $245.55 \pm 18.19$ & $243.55 \pm 14.08$ & $246.91 \pm 17.7$ & .675 \\
\hline \multirow[t]{2}{*}{ Thigh circumference $(\mathrm{cm})$} & 1st ERE & $53.72 \pm 1.34$ & $53.91 \pm 1.34$ & $54.03 \pm 1.30$ & $54.09 \pm 1.24$ & $54.03 \pm 1.31$ & $53.94 \pm 1.32$ & .999 \\
\hline & 2nd ERE & $53.55 \pm 1.32$ & $53.73 \pm 1.32$ & $53.71 \pm 1.33$ & $53.85 \pm 1.33$ & $53.7 \pm 1.29$ & $53.66 \pm 1.29$ & .999 \\
\hline \multirow[t]{2}{*}{ Muscle soreness (cm) } & 1st ERE & $1.18 \pm 0.38$ & $1.13 \pm 0.31$ & $1.61 \pm 0.35$ & $1.71 \pm 0.33$ & $1.42 \pm 0.34$ & $1.25 \pm 0.34$ & .796 \\
\hline & 2nd ERE & $1.00 \pm 0.27$ & $0.75 \pm 0.23$ & $1.22 \pm 0.31$ & $1.41 \pm 0.33$ & $1.01 \pm 0.34$ & $0.82 \pm 0.27$ & .615 \\
\hline
\end{tabular}

Values are means \pm SEM.

MVIC, maximal voluntary isometric contraction.

$p$-values are calculated by one-way analysis of variance.
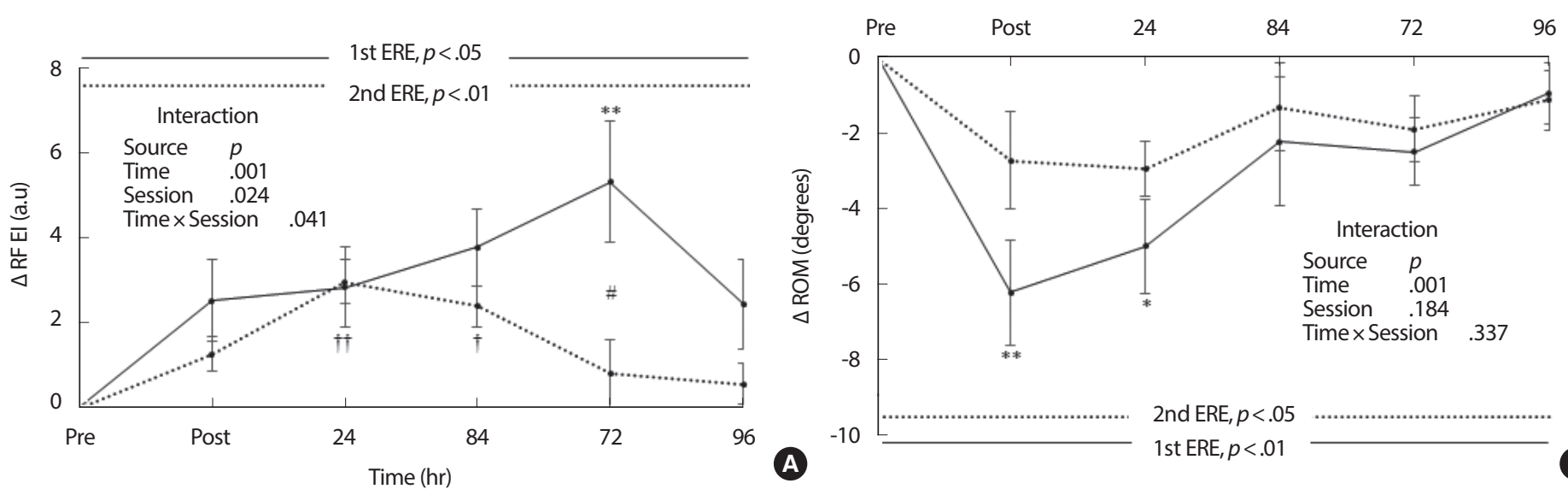

Fig. 2. Changes in muscle damage indicators after repeated bouts of eccentric exercise. (A) rectus femoris echo intensity; (B) range of motion of knee extensor. EIMD, exercise-induced muscle damage; RF, rectus femoris muscle; El, echo intensity; ROM, range of motion; ERE, eccentric resistance exercise. Values represent means \pm SEM. $p$ values are calculated with one-way analysis of variance (ANOVA). Interaction is examined with repeated measures ANOVA. ${ }^{*} p<.05$ and ${ }^{* *} p<.01$ indicate significant differences between baseline and each recovery period after the 1st eccentric exercise, analyzed with one-way ANOVA followed by Bonferroni's post hoc test. ${ }^{\dagger} p<.05$ and ${ }^{+\dagger} p<.01$ indicate significant differences between baseline and each recovery period after the 2 nd eccentric exercise, analyzed with one-way ANOVA followed by Bonferroni's post hoc test. ${ }^{\#} p<.05$ indicates a significant difference between the 1 st and 2 nd bouts, analyzed with Wilcoxon signed-rank test.

repeated bouts of ERE. Although there were significant differences in the changes in LF nu following each ERE bout (1st ERE, $p<.05 ; 2$ nd ERE, $p<.01)$, no significant interaction effects were observed $(p=.969)$ between the time and bout (Fig. 3A). HF nu gradually decreased after each bout of ERE and there were significant differences in the changes in HF nu from baseline following both experimental sessions (1st ERE, $p<.05 ; 2$ nd ERE, $p<.01$ ) (Fig. 3B). However, no significant interaction effects $(p=.969)$ between the time and session were observed on the changes in HF nu (Fig. 3B). The changes in the LF/HF ratio from baseline were statistically significant following both experimental trials $(p<.05)$ (Fig. 4). However, there were no significant interaction effects $(p=.510)$ between the time and session on changes in the LF/HF ratio (Fig. 4).

\section{DISCUSSION}

The present study was carried out to investigate HRV responses to acute ERE and to examine whether the CAR adapts to repeated bouts of ERE.

The main findings were as follows. Firstly, the maximal ERE of the knee extensor muscles influences muscle damage indicators and subsequent bouts following the initial exercise may provide a protective effect against EIMD caused by ERE. Secondly, in addition to sympathetic hyperactivity and vagal withdrawal in CAR following the maximal ERE, the cardiac sympathovagal balance was significantly shifted toward sympathetic predominance. Moreover, the cardiac autonomic imbalance induced by ERE was not adapted to repeated bouts of ERE.

It has been reported that the incidence and severity of EIMD caused by a single bout of ECC are lower in knee extensors, which experience 

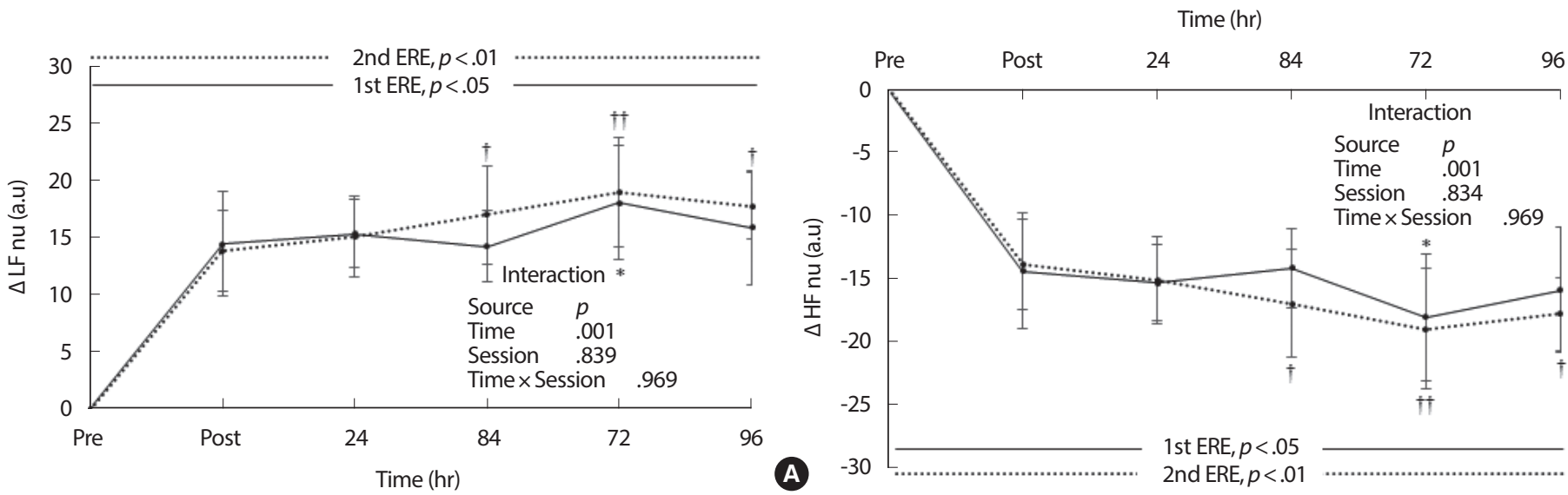

Fig. 3. Changes in normalized LF and HF after repeated bouts of eccentric exercise. (A) normalized low frequency; (B) normalized high frequency. LF nu, normalized low frequency $(0.04-0.15 \mathrm{~Hz}) ; \mathrm{HF}$ nu, normalized high frequency $(0.15-0.4 \mathrm{~Hz})$; ERE, eccentric resistance exercise. Values represent means $\pm \mathrm{SEM}$. $p$ values are calculated with one-way analysis of variance (ANOVA). Interaction is examined with repeated measures ANOVA. ${ }^{*} p<.05$ indicates significant differences between baseline and each recovery period after the 1st eccentric exercise, analyzed with one-way ANOVA followed by Bonferroni's post hoc test. ${ }^{\dagger} p<.05$ and ${ }^{+\dagger} p<.01$ indicate significant differences between baseline and each recovery period after the 2 nd eccentric exercise, analyzed with oneway ANOVA followed by Bonferroni's post hoc test.

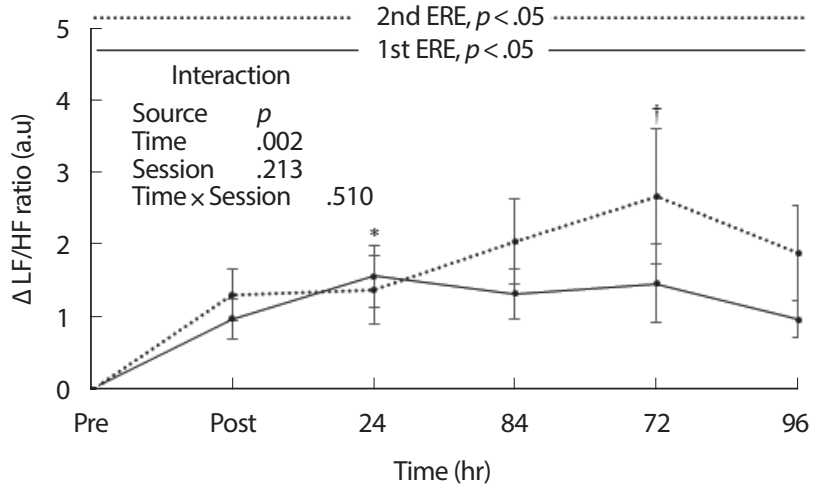

Fig. 4. Changes in LF/HF ratio after repeated bouts of eccentric exercise. LF/ $\mathrm{HF}$ ratio, ratio between $\mathrm{LF}(0.04-0.15 \mathrm{~Hz})$ and $\mathrm{HF}(0.15-0.4 \mathrm{~Hz})$ band powers; $E R E$, eccentric resistance exercise. Values represent means $\pm S E M$. $p$ values are calculated with one-way analysis of variance (ANOVA). Interaction is examined with repeated measures ANOVA. * $p<.05$ indicates significant differences between baseline and each recovery period after the 1st eccentric exercise, analyzed with one-way ANOVA followed by Bonferroni's post hoc test. ${ }^{\dagger} p<.05$ indicates significant differences between baseline and each recovery period after the 2 nd eccentric exercise, analyzed with one-way ANOVA followed by Bonferroni's post hoc test.

eccentric contractions during daily life activities, compared to elbow flexors, which are rarely exposed to eccentric action [19]. For these reasons, previous studies in which the investigators attempted to design an experimental model of muscle damage employed mainly ECC using elbow flexors rather than knee extensors $[7,20]$. However, knee extensors play a critical role in metabolic pathways and the locomotion system as the muscle groups with the largest cross-sectional area [21,22]. Furthermore, the damage and adaptation profiles of knee extensors following repeated bouts of ECC will provide useful clinical information to design resistance exercise (RE) programs, given that muscle damage such as sarcomeric disruption caused by RE is a physiological mechanism that leads to muscle hypertrophy [23]. The current study demonstrated that knee extensors such as the rectus femoris are damaged by the maximal ERE and that muscle EI is a valid tool to identify muscle damage alleviated by RBE. It has been documented in previous studies that muscle EI may reflect the RBE as well as the time-varying characteristics of EIMD after lengthening muscle contractions in the elbow flexors [24]. Consequently, this study indicated that maximal ERE may have led to EIMD of the rectus femoris, while repeated bouts of ERE were able to provide a protective effect on the knee extensor.

Acute RE can cause a greater delay in post-exercise parasympathetic reactivation than endurance exercise [25] and traditional RE decreases the LF nu of the HRV spectrum for up to 24 hours after exercise [26]. In contrast, the maximal ERE of the knee extensors significantly increased the LF nu or decreased the HF nu at 72 hours post-exercise in the present study. These results suggest that acute ERE may suppress the CAR for long periods compared to traditional RE, although the cardiovascular load during eccentric contraction is lower than that during concentric contraction [27]. Considering that ECC is recommended for elderly and patients with cardiovascular diseases because the cardiovascular demand can be alleviated during exercise [6], the results of the current study highlight the importance of performing cardiovascular monitoring for a sufficient length of time after acute ERE.

Chen et al. reported that acute RE led to cardiac autonomic imbalance despite being performed by weightlifters who had trained for 6 years or 
longer [26]. In the current study, changes in LF nu and HF nu lasted longer after a subsequent bout than after the initial ERE and the LF/HF ratio exhibited a greater increase after a subsequent bout than after the initial bout. Collectively, RBE after the maximal ERE as well as after the traditional RE did not appear to lead to cardiac autonomic adaptations. More surprisingly, a systematic review that examined the impact of RE on CAR after exercise concluded that post-exercise cardiac autonomic responses such as sympathetic hyperactivity and vagal withdrawal during recovery are more evident in resistance-trained men [2]. These authors suggested that increased catecholamine release is physiological factor that likely explains cardiac autonomic imbalance after acute RE [2]. However, data from previous work demonstrated that increased catecholamine levels caused by acute RE returned to the baseline levels within 1 hour after exercise [28]. Therefore, catecholamine activity does not explain cardiac autonomic imbalance, which can persist long after acute or repeated bouts of ERE. Meanwhile, EIMD symptoms following ECC may persist for several days [7] and there is a possibility that signs of muscle damage such as inflammatory processes [29] can be implicated in cardiac autonomic responses to acute ERE. Unfortunately, indirect indicators of EIMD employed in previous studies cannot explain cardiac autonomic imbalance after repeated bouts of ECC since most muscle damage indicators may be adapted to acute ECC via RBE [19]. However, to react more quickly to subsequent muscle damage, we should note that inflammatory responses may not be alleviated by repeated bouts of ECC [30], although the theory was not evaluated in this study. Microstructural damage induced by acute ERE may lead to an inflammatory response [29] and it has been suggested that the inflammatory process itself may be responsible for EIMD symptoms such as muscle soreness and swelling following ECC [19]. In addition, although it is not well understood why muscle EI increases after ECC, increased echogenicity may be caused by muscle conditions such as inflammatory myositis [31]. As increased inflammation can lead to decreased HRV [10,32], inflammatory responses to acute ERE may have an impact on both EIMD symptoms and CAR. However, the inflammatory theory has not been accepted as a valid mechanism to explain EIMD persisting for several days [33], since the changes in inflammatory markers recorded in previous studies decreased within 24 hours. Therefore, we believe that investigating valid inflammatory markers that can reveal EIMD symptoms is necessary, with a view to providing new insights into CAR as well as muscle damage following acute bouts of ERE.

The current study has several limitations. In accordance with clinical characteristics such as age and gender as well as diseases such as fibromyalgia and diabetes, which may have led to autonomic cardiac neuropathy, individual HRV responses to exercise may be very diverse. Although cardiac autonomic responses to acute RE indicate a consistent response defined as cardiac autonomic imbalance, independent of individual variation [2], it is not yet known how ERE will lead to changes in post-exercise cardiac autonomic control. Thus, only college-aged men were selected as an experimental population in the present study to eliminate elements that can influence cardiac autonomic responses after acute ERE. Since we investigated the changes in HRV indices after ERE, the results cannot be generalized to explain post-exercise cardiac autonomic responses to acute RE using different modalities. However, several studies have already demonstrated how traditional RE affects the CAR after exercise [2,26]. Consequently, the current study expanded our understanding of ERE, as it revealed that acute ERE compared to traditional RE can lead to cardiac autonomic imbalance persisting for a long time after exercise.

\section{CONFLICT OF INTEREST}

The authors have no conflict of interest to declare.

\section{AUTHOR CONTRIBUTIONS}

Data curation: C Kim, M Sun; Formal analysis: C Kim, M Sun; Methodology: C Kim, M Sun; Project administration: M Kim; Writing-original draft: C Kim, M Kim; Writing-review \& editing: M Kim.

\section{ORCID}

$\begin{array}{ll}\text { Maeng-Kyu Kim } & \text { https://orcid.org/0000-0002-7855-608X } \\ \text { Choun-Sub Kim } & \text { https://orcid.org/0000-0003-1995-080X } \\ \text { Min-Ghyu Sun } & \text { https://orcid.org/0000-0002-2487-9764 }\end{array}$

\section{REFERENCES}

1. Gielen S, Schuler G, Adams V. Cardiovascular effects of exercise training molecular mechanisms. Circulation. 2010;122(12):1221-38.

2. Kingsley JD, Figueroa A. Acute and training effects of resistance exercise on heart rate variability. Clin Physiol Funct I. 2016;36(3):179-87.

3. Melo RC, Quiterio RJ, Takahashi ACM, Silva E, Martins LEB, et al. 
High eccentric strength training reduces heart rate variability in healthy older men. Brit J Sport Med. 2008;42(1):59-63.

4. Kiviniemi AM, Hautala AJ, Karjalainen JJ, Piira OP, Lepojarvi S, et al. Acute post-exercise change in blood pressure and exercise training response in patients with coronary artery disease. Front Physiol. 2015;5 (526):1-7.

5. Douglas J, Pearson S, Ross A, McGuigan M. Chronic adaptations to eccentric training: a systematic review. sports Med. 2017;47(5):917-41.

6. Isner-Horobeti ME, Dufour SP, Vautravers P, Geny B, Coudeyre E, et al. Eccentric exercise training: modalities, applications and perspectives. Sports Med. 2013;43(6):483-512.

7. Chen TCC, Chen HL, Pearce AJ, Nosaka K. Attenuation of eccentric exercise-induced muscle damage by preconditioning exercises. Med Sci Sport Exer. 2012;44(11):2090-8.

8. Lindstedt SL, LaStayo PC, Reich TE. When active muscles lengthen: Properties and consequences of eccentric contractions. News Physiol Sci. 2001;16(6):256-61.

9. Chen TC, Lin KY, Chen HL, Lin MJ, Nosaka K. Comparison in eccentric exercise-induced muscle damage among four limb muscles. Eur J Appl Physiol. 2011;111(2):211-23.

10. Thayer JF, Yamamoto SS, Brosschot, JF. The relationship of autonomic imbalance, heart rate variability and cardiovascular disease risk factors. Int J Cardiol. 2010;141(2):122-31.

11. Jouven X, Empana JP, Schwartz PJ, Desnos M, Courbon D, et al. Heart-rate profile during exercise as a predictor of sudden death. New Engl J Med. 2005;352(19):1951-8.

12. Faul F, Erdfelder E, Buchner A, Lang AG. Statistical power analyses using $\mathrm{G}^{\star}$ Power 3.1: tests for correlation and regression analyses. Behav Res Methods. 2009;41(4):1149-60.

13. Wilhelm EN, Rech A, Minozzo F, Radaelli R, Botton CE, et al. Relationship between quadriceps femoris echo intensity, muscle power, and functional capacity of older men. Age. 2014;36(3):1113-22.

14. Medeiros DM, Mantovani RF, Lima CS. Effects of low-intensity pulsed ultrasound on muscle thickness and echo intensity of the elbow flexors following exercise-induced muscle damage. Sport Sciences for Health. 2017;13(2):365-71.

15. Hawes MR, Martin AD. Human body composition. Kinanthropometry and exercise physiology laboratory manual: tests, procedures and data 2001;1:7-46

16. Ormsbee MJ, Ward EG, Bach CW, Arciero PJ, McKune AJ, et al. The impact of a pre-loaded multi-ingredient performance supplement on muscle soreness and performance following downhill running. J Int Soc Sports Nutr. 2015;12(2):1-9.

17. Task Force of the European Society of Cardiology. Heart rate variability: standards of measurement, physiological interpretation and clinical use. Circulation. 1996;93(5):1043-65.

18. Kim CS, Kim MK, Jung HY, Kim MJ. Effects of exercise training intensity on cardiac autonomic regulation in habitual smokers. Ann Noninvasive Electrocardiol. 2017;22(5):e12434.

19. Hyldahl RD, Chen TC, Nosaka K. Mechanisms and mediators of the skeletal muscle repeated bout effect. Exerc Sport Sci Rev. 2017;45 (1):24-33

20. Nosaka K, Sakamoto K, Newton M, Sacco P. The repeated bout effect of reduced-load eccentric exercise on elbow flexor muscle damage. Eur J Appl Physiol. 2001;85(1-2):34-40.

21. Elder CP, Apple DF, Bickel CS, Meyer RA, Dudley GA. Intramuscular fat and glucose tolerance after spinal cord injury-a cross-sectional study. Spinal Cord. 2004;42(12):711-6.

22. Lamy JC, Iglesias C, Lackmy A, Nielsen JB, Katz R, et al. Modulation of recurrent inhibition from knee extensors to ankle motoneurones during human walking. J Physiol-London. 2008;586(24):5931-46.

23. Kruger M, Kotter S. Titin, a central mediator for hypertrophic signaling, exercise-induced mechanosignaling and skeletal muscle remodeling. Front Physiol. 2016;7(76):1-8.

24. Chen TC, Chen HL, Lin MJ, Wu CJ, Nosaka K. Muscle damage responses of the elbow flexors to four maximal eccentric exercise bouts performed every 4 weeks. Eur J Appl Physiol. 2009;106(2):267-75.

25. Heffernan KS, Rossow L, Jae SY, Shokunbi HG, Gibson EM, et al. Effect of single-leg resistance exercise on regional arterial stiffness. Eur J Appl Physiol. 2006;98(2):185-90.

26. Chen JL, Yeh DP, Lee JP, Chen CY, Huang CY, et al. Parasympathetic nervous activity mirrors recovery status in weightlifting performance after training. J Strength Cond Res. 2011;25(6):1546-52.

27. Okamoto T, Masuhara M, Ikuta K. Cardiovascular responses induced during high-intensity eccentric and concentric isokinetic muscle contraction in healthy young adults. Clin Physiol Funct I. 2006;26(1):3944.

28. Kliszczewicz BM, Esco MR, Quindry JC, Blessing DL, Oliver GD, et al Autonomic responses to an acute bout of high-intensity body weight resistance exercise vs. treadmill running. J Strength Cond Res. 2016;30(4) :1050-8.

29. Peake J, Nosaka K, Suzuki K. Characterization of inflammatory re- 
sponses to eccentric exercise in humans. Exerc Immunol Rev. 2005; 11:64-85.

30. Deyhle MR, Gier AM, Evans KC, Eggett DL, Nelson WB, et al. Skeletal muscle inflammation following repeated bouts of lengthening contractions in humans. Front Physiol. 2016;6(424):1-11.

31. Campbell SE, Adler R, Sofka CM. Ultrasound of muscle abnormali- ties. Ultrasound Quarterly. 2005;21(2):87-94.

32. Haensel A, Mills PJ, Nelesen RA, Ziegler MG, Dimsdale JE. The relationship between heart rate variability and inflammatory markers in cardiovascular diseases. Psychoneuroendocrino. 2008;33(10):1305-12.

33. Cheung K, Hume PA, Maxwell L. Delayed onset muscle soreness. Sports Med. 2003;33(2):145-64. 